\title{
Identification of biomarkers and enriched pathways involved in lung cancer
}

\author{
Nikita Singh $^{1}$, Mukesh Kumar ${ }^{2 *}$, Atanu Bhattacharjee ${ }^{3}$, Prashant Kumar Sonker ${ }^{4}$, Agni Saroj ${ }^{4}$ \\ ${ }^{1}$ Department of Bioinformatics, M.M.V., Banaras Hindu University, Varanasi, India \\ ${ }^{2}$ Department of Statistics, M.M.V., Banaras Hindu University, Varanasi, India \\ ${ }^{3}$ Section of Biostatistics, Centre for Cancer Epidemiology, TMC Mumbai, India \\ ${ }^{4}$ Department of Statistics, I. Sc., Banaras Hindu University, Varanasi, India
}

*Corresponding Author:
Mukesh Kumar, PhD
Department of Statistics, MMV
Banaras Hindu University
Varanasi-221005
Email: mukesh.mmv@bhu.ac.in

\begin{abstract}
Objective: The aim of study is to find key genes and enriched pathways associated with lung cancer.
\end{abstract}

Participants and Methods: Differentially expressed genes (DEGs) data of 54674 genes based on stage, tumor and status of lung cancer was taken from 66 patients of African American (AAs) origin. 2392 DEGs were found based on stage, 13502 DEGs were found based on tumor, 2927 DEGs were found based on status having $\mathrm{p}$ value $(\mathrm{p}<0.05)$.

Results: Total 33 common DEGs were found from stage, tumor and status of lung cancer. Gene ontology (GO) and KEGG pathway enrichment analysis was performed and 49 significant pathways were obtained, out of which 10 pathways were found to be exclusively involved in lung cancer development. Protein-protein interaction (PPI) network analysis found 69 nodes and 324 edges and identified 10 hub genes based on their highest degrees. Module analysis of PPI found that 'Viral carcinogenesis', 'pathways in cancer', 'notch signaling pathway', 'AMPK signaling pathways' had a close association with lung cancer.

Conclusion: These identified DEGs regulate other genes which play important role in growth of lung cancer. The key genes and enriched pathways identified can thus help in better identification and prediction of lung cancer.

\section{Keywords}

Lung cancer, biomarker, gene ontology, protein-protein interaction networks, survival analysis 


\section{Introduction}

Worldwide mortality from lung cancer growth expanded from 3.5 million in 1990 to 4.2 million in $2015^{1}$ and it is assessed that there will be 2.1 million new lung cancer incidents and 1.8 million deaths in 2018, representing (18.4\%) incidents of cancer-related mortality. ${ }^{2}$ Lung cancer is a heterogeneous disease and various factors including hereditary transformations, ecological components and individual habits can add to cancer incident, evolution and metastasis. ${ }^{3}$ According to histological disparity, lung cancer can be partitioned into non-small cell lung cancer (NSCLC) and small-cell lung cancer (SCLC), of which NSCLC representsroughly85\%, and $30 \%$ of SCLC cases can be named as lung squamous cell carcinoma. ${ }^{4}$ It is reported that a number of genes and biological, cellular and molecular pathways take part in these processes. Hence, it is crucial to understand the important mechanisms that lead to the onset and development of lung cancer in order to produce diagnostic and therapeutic strategies. A past researches on gene expression profiling in cancer used microarray tools for examining oncology ${ }^{5}$ however some of these studies have been directed on lung cancer with comparative analysis of the DEGs ${ }^{6}$, and a very authentic biomarker profile refining cancerous tissues from normal ones remains to be discovered.

In the present study, gene expression data of mRNAs and miRNAs have been taken from 66 patients of AAs origin. A total of 54674 genes were screened, on the basis of stage (I or II), tumor (present or absent) and status (dead or alive). Student's t-test for difference of means assuming unequal variances was applied to test the datasets and two-tailed $(\mathrm{p}<0.05)$ was considered statistically significant. Out of these, 2392 DEGs from stage of lung cancer, 13502 DEGs from tumor and 2979 DEGs from status were obtained. 33 common DEGs from stage, tumor and status of the lung cancer were found. These 33 DEGs were screened further for gene ontology (GO) using DAVID database. The genes were analysed in STRING database for showing PPI network analysis. KEGG pathway analysis was also performed to see the pathway enriched among these genes. Using Cytoscape software, PPI was visualized. Using MCODE plug-in of Cytoscape, module analysis was performed and top 3 modules involved in lung cancer were identified which depicted top 6 pathways with genes involved in them. Using CYTOHUBBA plug-in of Cytoscape, top 10 hub genes involved in the lung cancer were identified along with their respective ranks and score. In survival analysis, Kaplan-Meier (KM) curve was drawn to represent the survival of lung cancer patients. The aim of this study is to find DEGs and related pathways for development of lung cancer and also identify possible genes 
biomarkers for identification and prospects of lung cancer.

\section{Materials and methods}

Gene expression data: The mRNA and miRNA of 66 patients of AAs origin was used for analysis purpose. The DEGs data was based on stage, tumor and status of lung cancer patients. Data is obtained from gene omnibus website https://www.ncbi.nlm.nih.gov/geo/. The accession number of the data is GSE102287.

Student's t-test for identification of significant genes: We have taken 54647 DEGs in 66 patients of AAs origin. We have categorized the genes on the basis of stage, tumor and status. Student's t-test is applied to test the difference of means for unequal variances on the basis of $\mathrm{p}$ value. This procedure is adopted to screen the gene expression data and find out the DEGs based on their $(\mathrm{p}<0.05)$ Table 2.

Heat map: Heat map is used to represent the level of expression genes with comparable samples. By using $\mathrm{R}$ software we have created heat maps to show gene expressions level for DEGs obtained based on stage 1 and stage 2. Thereafter the patients were classified as tumor present, tumor absent and dead and alive status. Now the gene expression levels are shown by yellow, orange and red colors with gene affy ID and patients ID along with $x$-axis and y-axis respectively.

GO term enrichment analysis: GO of these 205 DEGs were done using DAVID Database that is available at https://david.ncifcrf.gov/.GO is a major bioinformatics activity to combine the demonstration of gene and gene product attributes with all variety. The aim is to: 1) maintain and expand its restricted vocabulary of gene and gene product attributes; 2) interpret genes and gene products data; and 3) provide tools for easy access to all aspects of the data. ${ }^{7}$

Establishment of PPI Network: Search Tool for Retrieval of the Interacting genes(String) online database is used for representation of PPI networks and available at https://string-db.org/ . A frame work comprehension of cell function requires information of all practical relations between the expressed proteins. The STRING database is used to collect and combine this information and predicted Protein-Protein Interaction (PPI) for a large number of organisms. ${ }^{8}$ Investigating the predicted interaction networks can recommend new directions for future computational research and provide cross-species expectations to efficient associated mapping. ${ }^{9}$ String database gave the list of most significantly enriched pathways by KEGG pathway analysis. In the PPI network, the nodes involved in pathways exclusively involved in lung cancer 
with various colors were highlighted. These pathways showed genes that were involved in the NSCLC with their false discovery rates.

Cytoscape: This is online open software platform for representation molecular communication networks and genetic pathways and combine these networks with annotations, gene expression profiles and other state of data and can be downloaded from https://cytoscape.org/. Cytoscapeis used to provide a basic set of features for data integration, analysis, and representation. The string file was saved in .tsv format and was imported in Cytoscape software. Using the MCODE (molecular complex detection) plug-in of Cytoscape, top 3 modules of protein-protein interactions were visualized that are seen to be involved in the lung cancer. By using CYTOHUBBA plug-in of cytoscape, we found top 10 hub genes which are highly involved in lung cancer.

TCGA database: TCGA database offers various computational tools that can be used to analyze data. One such tool is cBioPortal for cancer genomics (http://www.cbioportal.org/). The cBioPortal for Cancer Genomics provides visualization, analysis and download of large-scale cancer genomics data sets. This tool was used to find the role of hub genes in NSCLC. Oncoprint and cancer type summary was studied for all the hub genes.

Survival analysis: Survival analysis is used to analysis of life time until one or more event happen. The KM curve is used to estimate the survival of patients from time dependent data. In medical sciences, it is often used to find the fraction of patients living for a certain time after treatment. Here, we have plotted the KM curve using R software for the stage-wise survival of lung cancer patients. ${ }^{23}$

\section{Results}

After applying student's t-test for unequal variances on 54647 genes with their gene expression values, we obtained 33 common DEGs. The selected genes had $(\mathrm{p}<0.05)$ in (Table 2). The description of cancer patients is shown in (Table 1).

A total of 6 heat maps were plotted to show gene expressions level for DEGs obtained based on stage 1 and stage 2, tumor present and absent, and dead status and alive status. The yellow color in the heat map indicates lower values of gene expression values while the orange ones are intermediate and red ones have the highest values which had high values for the gene expression (Figure 1).To find the role of the DEGs, GO term enrichment analysis was performed with 
online database DAVID. The genes were significantly enriched in biological process (BP), molecular function (MF) and cellular component (CC) (Table 3). The genes were enriched significantly in BP, including 'directive of receptor activity', 'Anterior/posterior pattern specification'. The genes enriched in MF, including 'Poly (A) RNA binding' and 'Protein binding'. KEGG pathway analysis is used to identify the pathways involving these genes. A total of 24 significantly enriched pathways were identified (Table 4). The most significantly enriched pathways related with lung cancer were 'AMPK signaling pathway', 'PPAR signaling pathway', 'pathways in cancer', 'PI3K-Akt signaling pathway', 'notch signaling pathway', 'viral carcinogenesis', 'microRNAs in cancer', 'HIF-1 signaling pathway', 'Valine, leucine and isoleucine degradation' and 'Wnt signaling pathway' (Figure 2 and Table 5).The PPI network is constructed to classify the mainly important proteins and genetic modules that may serve critical roles in the growth of lung cancer. A total of 69 nodes and 324 edges were screened from PPI network (Figure 2). The average node degree was 9.39, the average local coefficient clustering was 0.694 and the PPI enrichment $(\mathrm{p}<0.01)$. Each gene was entrusted a degree that predicted number of adjacent nodes in the network and changes in proteins/genes. The top 10 hub genes with the highest degrees in lung cancer were EP300 (E1A Binding Protein P300), TP53 (Tumor protein 53), KAT2B(lysine acetyltransferase2B), HDAC1 (Histone Deacetylase 1), SIRT1 (sirtuin 1), KMT2A (Lysine Methyltransferase 2A), ASH2L (histone lysine methyltransferase complex subunit), SETD1B (SET Domain Containing 1B), SETD1A (SET Domain Containing 1A), KMT2C (Lysine Methyltransferase 2C) (Table 6 and Figure 3). EP300 has highest degree of 29. It is found that high degree of these hub genes which play animportant role in maintaining the entirePPI. In addition, to find the significance DEGs, the top 3 significant modules were selected and functional interpretation of genes related with the modules were analyzed (Figure 4 and Table 7). The results described that these modules had pathways that were seen to play a critical role in lung cancer. Module 1 was associated with viral carcinogenesis, pathways in cancer, notch signaling pathway, microRNAs in cancer, wnt signaling pathway. Module 2 was associated with AMPK signaling pathway, PPAR signaling pathway, PI3K-Akt signaling pathway, HIF-1 signaling pathway. Module 3 was associated with AMPK signaling pathway, pathways in cancer, wnt signaling pathway. cBioPortal is a computational tool present in TCGA database that provides representation, analysis and download of cancer DEGs data. This tool is used to evaluate the oncoprint (Figure 5), lung cancer type summary which depicts the mutations, fusion, amplification etc in the genes. It was found that out of 10 hub genes, only 4 
were exclusively involved in lung cancer EP300, TP53, KMT2A and KMT2C. The 4 genes underwent mutations largely.KM plotted for the stage-wise survival curves of lung cancer AAs patients. Stage 3 clearly depicts the lowest rate of survival among all the 3 stages (Figure 6).

\section{Discussion}

Cancer is basically a hereditary disease, and different hereditary changes collect during the multistep process of carcinogenesis, which finally leads to anomalous excessive cell development and malignant phenotype. ${ }^{10}$ Lung cancer is basically essential pulmonary malignant tumor in terms of incidence and mortality. ${ }^{11}$ Early identification and efficient treatment of lung cancer is need of the hour and it can be achieved by the identification of significant genes and understanding their molecular mechanisms which play an important role in causing lung cancer. DEGs data of various genes can be used for further functional analysis and to screen biomarkers that can serve for early identification and remedial targets. Therefore, they may help in finding of lung cancer in the early stages and can be used for the development of targeted treatment.

In present study statistical and bioinformatics methods are applied to identify new candidate genes that can serve critical roles in development of lung cancer. The data used here has gene expression values of 54674 genes for 66 patients, being categorized on the basis of stage, tumor and status of the lung cancer. A total of 33 common DEGs from stage, tumor and status were obtained based on their p-value score calculated by t-test for difference of means with unequal variances. Then, GO and KEGG pathway analyses are performed to find the associations of these significant genes. Finally, a PPI network was constructed that depicted that these identified DEGs directly do not play role in causing lung cancer, but they interact and regulate other neighboring genes that play a very important role in development of lung cancer (Figure 2).

GO analysis is helpful for annotating genes and gene products. GO analysis in the present study showed that these significant genes involved in biological process like 'Regulation of receptor activity', Anterior/posterior pattern specification', molecular functions like 'Poly (A) RNA binding' and 'Proteinbinding'. It is observed that defective functioning of biological processes and body system status are important causes of tumor growth and evolution. Hence, monitoring the expression of these genes may help in discovery of tumor mechanisms. The KEGG pathway database carries methodical analysis of gene functions, linking genomics and the functional information. Enrichment analysis is used to find important and most significant 
KEGG pathways which are related with lung cancer and its growth were 'AMPK signaling pathway', 'PPAR signaling pathway', 'pathways in cancer', 'PI3K-Akt signaling pathway', 'notch signaling pathway', 'viral carcinogenesis', 'microRNAs in cancer', 'HIF-1 signaling pathway', 'Valine, leucine and is oleucine degradation' and 'Wnt signaling pathway' (Figure 2 and Table 5).Taking pathways into consideration, AMPK plays a central role in the control of cell growth, prevalence and autotrophic through the rule of mTOR activity, which is consistently uncontrolled in cancer cells. Targeting of AMPK/mTOR is thus strategy in the growth of remedial elements against NSCLC. ${ }^{12}$ The PI3K pathway is frequently uncontrolled in lung. ${ }^{13}$ Cancer due to hereditary variation affecting its components resulting in increased PI3K signaling PPAR- $\gamma$ factor bring development and promote changes related with separation as well as apoptosis in different lung carcinoma cell lines. ${ }^{14}$ Thus, defects in PPAR signaling pathway can promote tumor growth. In case of notch signaling pathway and Dang et al. found that the over expression of Notch3 was perceived in $40 \%$ of patients with NSCLC, and that this over expression was connected with a translocation including $19 \mathrm{p} .{ }^{15}$ In HIF-1 signaling pathway, Hypoxia-inducible factor- $1 \alpha$ (HIF-1 $\alpha$ ) is over expressed in human lung diseases, especially in NSCLC, and is firmly related with a propelled tumor grade, expanded angiogenesis, and protection from chemotherapy and radiotherapy. ${ }^{16}$ In case of wnt signaling pathway, over expression of Wnt-1, -2, -3, and -5a and of Wnt-pathway components Frizzled-8, Disheveled, Porcupine, and TCF-4 is common in NSCLC and is associated with poor prognosis. ${ }^{17}$ p53 is the most frequently mutated gene in lung cancer. ${ }^{18}$ Most clinical studies suggest that NSCLC with TP53 alterations carries a worse prognosis and may be relatively more resistant to chemotherapy and radiation. ${ }^{19}$ Inactivation of TP53 capacity or its orderly pathway is a typical component of human tumors that regularly relates with expanded danger, poor patient survival, and protection from treatment. ${ }^{20-22}$

It is observed that many genes though not in our 33 common DEGs, comes into picture because it is regulated by genes present in our initial DEGs list such as PPP1R3C, ACAA2, TRIM5, PCSK9, P2RY1, CISH, PARN and KMT2A (Figure 2). Hence, it is clearly seen that the 33 DEGs do not directly participate in development of lung cancer but some of them influence and regulate other genes which play key role in development of lung cancer. PPP13RC is predicted functional partner of GYS1 AND GYS2. ACAA2 is neighbor of ACADM. TRIM5 and PCSK9 are in a cluster network of APOA1 and APOA2. P2Y1 is connected to CREB1. CISH is connected to two most crucial genes TP53 and EP300. PARN is found associated with TP53. 
KMT2A is the gene with high no. of degree among our 33 DEGs. It is connected to CREB1, EP300, TP53, HDAC1 and SIRT1.

The string file is imported in cytoscape software and using CYTOHUBBA plug-in, top 10 hub genes based on their degree was found. The gene with highest score was EP300, followed by TP53 and KAT2B (Figure 3 and Table 6). These 10 hub genes played important role in growth of lung cancer. Using MCODE plug-in of cytoscape, top 3 modules of this network were seen which were again observed to take part in pathways that caused lung cancer (Figure 4 and Table 7). The oncoprint and cancer summary type study is done by cBioPortal of TCGA database shows that TP53 is most mutated gene among all the top 10 hub genes. Also among the 10 hub genes, only 4 genes are exclusively involved in lung cancer viz. EP300, TP53, KMT2C and KMT2A. Cancer type summary is depicted in figure 6. The survival analysis was done and KM Plot was plotted which demonstrated that Stage 3 clearly has the lowest rate of survival among all the 3 stages.

Hence, this study made us to reach on a conclusion that DEGs may directly be involved in the pathways that lead to the development of cancer or may sometimes be indirectly involved like influencing and regulating other genes and their pathways that may play a crucial role in development of a tumor or a cancer.

\section{Conclusion}

Overall, through identification and functional analysis of DEGs we identified Regulation of receptor activity, anterior/posterior pattern specification and protein binding as significant terms for lung cancer. The initial 33 DEGs found in this study trigger or influence other neighboring DEGs that may be directly involved in the onset or development of lung cancer. Although, it may be early to suggest that these DEGs might be ready for clinical trials, it is clearly a direction that wants further attention. All these results may help us in better diagnosis and prognosis of lung cancer and may pave way for better treatment of the disease.

\section{Ethical statements:}

The current study has been done based on secondary data source. Data is obtained from gene omnibus website https://www.ncbi.nlm.nih.gov/geo/. There is no need of ethical approval for this study.

Funding: The authors have no received any funding for this research work. 
Conflicts of Interest: The authors declare no conflict of interest.

Author Contributions: All authors had full access to the data in the study and take responsibility for the integrity of the data and the accuracy of the data analysis. Conceptualization, MK.; Formal Analysis, NS, PKS, AS; Resources, AB; Writing-Original Draft, NS.

\section{References}

1. Cohen AJ, Brauer M, Burnett R, Anderson HR, Frostad J, Estep K, Balakrishnan K, Brunekreef B, Dandona L, Dandona R, et al: Estimates and 25-year trends of the global burden of disease attributable to ambient air pollution: An analysis of data from the Global Burden of Diseases Study 2015. Lancet 389: 1907-1918, 2017.

2. Bray F, Ferlay J, Soerjomataram I, Siegel RL, Torre LA and Jemal A: Global cancer statistics 2018: GLOBOCAN estimates of incidence and mortality worldwide for 36 cancers in 185 countries. CA Cancer J Clin 68: 394-424, 2018.

3. Aran V, Victorino AP, Thuler LC and Ferreira CG: Colorectal cancer: Epidemiology, disease mechanisms and interventions to reduce onset and mortality. Clin Colorectal Cancer 15: 195-203, 2016.

4. Piperdi, B, Merla, A, \& Perez-Soler, R. Targeting angiogenesis in squamous non-small cell lung cancer. Drugs, 74(4), 403-413(2014).

5. Descotes F, Dessen P, Bringuier PP, Decaussin M, Martin PM, Adams M, Villers A, Lechevallier E, Rebillard X, Rodriguez-Lafrasse C, et al: Microarray gene expression profiling and analysis of bladder cancer supports the sub-classification of T1 tumours into T1a and T1b stages. BJU Int 113: 333-342, 2014.

6. Sakashita H, Inoue H, Akamine S, Ishida T, Inase N, Shirao K, Mori M and Mimori K: Identification of the NEDD4L gene as a prognostic marker by integrated microarray analysis of copy number and gene expression profiling in non-small cell lung cancer. Ann SurgOncol 20 (Suppl 3): S590-S598, 2013.

7. The Gene Ontology Consortium, Michael Ashburner, Catherine A. Ball, Judith A. Blake, David Botstein, Heather Butler, J. Michael Cherry, Allan P. Davis, Kara Dolinski, Selina S. Dwight, Janan T. Eppig, Midori A. Harris,David P. Hill, Laurie Issel-Tarver, Andrew Kasarskis, Suzanna Lewis, John C. Matese, Joel E. Richardson, Martin Ringwald,Gerald M. Rubin, and Gavin Sherlock, "Gene Ontology: tool for the unification of biology", HHS author manuscript, PMC3037419.

8. Damian Szklarczyk, John H Morris, Helen Cook, Michael Kuhn, Stefan Wyder, Milan Simonovic, Alberto Santos, Nadezhda T Doncheva,Alexander Roth, Peer Bork, Lars J. Jensen, and Christian von Mering, "The STRING database in 2017 : quality-controlled protein-protein interaction networks, made broadly accessible", Nucleic acid research, vol. 45(database issue), 2014 jan 4.

9. Schwartz, AS; Yu, J; Gardenour, KR; Finley Jr; RL \&Ideker, T : “Cost-effective strategies for completing the interactome"(2018). 
10.Carter SL, Eklund AC, Kohane IS, Harris LN and Szallasi Z: A signature of chromosomal instability inferred from gene expression profiles predicts clinical outcome in multiple human cancers. Nat Genet 38: 1043-1048, 2006.

11.Dela Cruz CS, Tanoue LT and Matthay RA: Lung cancer: Epidemiology, etiology, and prevention. Clin Chest Med 32: 605-644, 2011.

12.Han Dong; Li, Shao-Jun; Zhu, Yan-Ting; Liu, Lu; Li, Man-Xiang: LKB1/AMPK/mTOR Signaling Pathway in Non-small-cell Lung Cancer, Asian pacific journal of cancer prevention, 2013; vol.14 (issue 7).

13.Duronio V. The life of a cell: Apoptosis regulation by the PI3K/PKB pathway. Biochem. J. 2008;415:333-344.

14.Han S, Roman J. Peroxisome proliferators-activated receptor gamma: a novel target for cancer therapeutics? Anticancer Drugs. 2007;18:237-244.

15.Dang TP, Gazdar AF, Virmani AK, Sepetavec T, Hande KR, Minna JD, Roberts JR, Carbone DP. Chromosome 19 translocation, over expression of Notch3, and human lung cancer. J Natl Cancer Inst. 2000;92:1355-1357.

16.Qunzhou Zhang, Xudong Tang, Zuo-Feng Zhang, Rita Velikina, Shihong Shi and Anh D. Le"Nicotine Induces Hypoxia-Inducible Factor-1 $\alpha$ Expression in Human Lung Cancer Cells via Nicotinic Acetylcholine Receptor-Mediated Signaling Pathways". Clinical cancer research (2007).

17.Stewart DJ: "Wnt signaling pathway in non-small cell lung cancer". J Natl Cancer Inst. 2014 Jan;106(1):djt356.

18. Olivier M, Petitjean A, Marcel V, et al. Recent advances in p53 research: an interdisciplinary perspective. Cancer Gene Ther. 2009; 16:1-12.

19. Akira Mogi and Hiroyuki Kuwano (December 2010), "TP53 Mutations in Nonsmall Cell Lung Cancer”, J Biomed Biotechnology, vol. 2011, 2011.

20. A. J. Munro, S. Lain, and D. P. Lane, "P53 abnormalities and outcomes in colorectal cancer: a systematic review," British Journal of Cancer, vol. 92, no. 3, pp. 434-444, 2005.

21. B. Vogelstein, D. Lane, and A. J. Levine, "Surfing the p53 network," Nature, vol. 16, pp. 307-310, 2000.

22. K. H. Vousden and X. Lu, "Live or let die: the cell's response to p53," Nature Reviews Cancer, vol. 2, no. 8, pp. 594-604, 2002.

23.Kumar M, Sonker PK, Saroj A, Jain A, Bhattacharjee A, Saroj RK. Parametric survivalanalysis using R: Illustration with lung cancer data. Cancer Reports. 2019; 1210. https://doi.org/10.1002/cnr2.1210. 
Table 1: Summary of cancer patients involved in study

\begin{tabular}{|l|c|}
\hline \multicolumn{1}{|c|}{ Variable } & Number (\%) \\
\hline Total number of patients & 66 \\
\hline Age, median (range) (years) & $60(32-76)$ \\
\hline Male sex & $38(57.5 \%)$ \\
\hline Female sex & $28(42.4 \%)$ \\
\hline Smoking history & $41.47(62 \%)$ \\
\hline Cancer stages & $36(54.5 \%)$ \\
Stage I & $30(45.4 \%)$ \\
Stage II and III & \\
\hline Tumor & $32(48.4 \%)$ \\
Present & $34(51.1 \%)$ \\
Absent & \\
\hline Status & $26(39.3 \%)$ \\
Dead & $40(60.6 \%)$ \\
Alive & \\
\hline
\end{tabular}

Table 2: Common DEGs based on Status, tumor and status of lung cancer

\begin{tabular}{|c|c|c|c|c|}
\hline Gene Symbol & Mean & $\begin{array}{l}\text { Standard } \\
\text { Deviation }\end{array}$ & P-Value & Gene Description \\
\hline NKTR & 5.43 & 0.54 & 0.01 & Natural killer cell triggering receptor \\
\hline C12orf80 & 1.93 & 0.04 & 0.02 & $\begin{array}{c}\text { Chromosome } 12 \text { open reading frame } \\
80\end{array}$ \\
\hline LOC101927406 & 2.57 & 0.06 & 0.04 & Uncharacterized LOC101927406 \\
\hline ACAA2 & 3.63 & 0.38 & 0.04 & Acetyl-CoA acyltransferase \\
\hline GPX2 & 3.84 & 3.66 & 0.01 & Glutathione peroxidase \\
\hline PARN & 9.39 & 0.35 & 0.02 & Poly(A)-specific ribonuclease \\
\hline PPP1R3C & 8.42 & 1.77 & 0.01 & $\begin{array}{c}\text { Protein phosphatase } 1 \text { regulatory } \\
\text { subunit } 3 \mathrm{C}\end{array}$ \\
\hline S100P & 9.99 & 2.88 & 0.03 & S100 calcium binding protein $\mathrm{P}$ \\
\hline HOXC5 & 2.04 & 0.05 & 0.03 & Homeobox C5 \\
\hline P2RY1 & 4.20 & 0.88 & 0.00 & Purinergic receptor P2Y1 \\
\hline KLF12 & 2.14 & 0.03 & 0.04 & Kruppel like factor \\
\hline TRANK1 & 7.65 & 0.64 & 0.03 & $\begin{array}{c}\text { Tetratricopeptide repeat and ankyrin } \\
\text { repeat containing } 1\end{array}$ \\
\hline FXYD5 & 10.92 & 0.56 & 0.01 & $\begin{array}{l}\text { FXYD domain containing ion } \\
\text { transport regulator } 5\end{array}$ \\
\hline TRIM5 & 2.88 & 0.40 & 0.02 & Tripartite motif containing 5 \\
\hline $\mathrm{CISH}$ & 8.38 & 0.95 & 0.04 & $\begin{array}{c}\text { Cytokine inducible } \mathrm{SH} 2 \text { containing } \\
\text { protein }\end{array}$ \\
\hline PCSK9 & 5.45 & 0.93 & 0.01 & $\begin{array}{c}\text { Proproteinconvertasesubtilisin/ kexin } \\
\text { type } 9\end{array}$ \\
\hline
\end{tabular}




\begin{tabular}{|c|c|c|c|c|}
\hline SLIT1-AS1 & 2.18 & 0.04 & 0.01 & SLIT1 antisense RNA 1 \\
\hline TFB1M & 4.24 & 0.41 & 0.00 & $\begin{array}{c}\text { Transcriptional factor B1, } \\
\text { mitochondrial }\end{array}$ \\
\hline $\begin{array}{c}\text { SLX1A- } \\
\text { SULT1A3 }\end{array}$ & 7.12 & 0.48 & 0.03 & $\begin{array}{c}\text { SLX1A-SULT1A3 readthrough (NMD } \\
\text { Candidate) }\end{array}$ \\
\hline CC2D2A & 4.43 & 0.61 & 0.02 & $\begin{array}{c}\text { Coiled-coil and C2 containing domain } \\
\text { 2A }\end{array}$ \\
\hline LOC100507277 & 1.87 & 0.04 & 0.04 & Uncharacterized LOC100507277 \\
\hline FAM118B & 3.78 & 0.38 & 0.00 & $\begin{array}{c}\text { Family with sequence similarity 118 } \\
\text { member B }\end{array}$ \\
\hline KMT2A & 6.03 & 0.67 & 0.02 & Lysine methyltransferase 2A \\
\hline LOC100130502 & 1.78 & 0.06 & 0.03 & Uncharacterized LOC100130502 \\
\hline
\end{tabular}

Table 3: Gene Ontology analysis of DEGs

\begin{tabular}{|c|c|c|c|c|}
\hline S.NO. & CATEGORY & TERM & GENE COUNT & P-VALUE \\
\hline 1. & GO_BP_DIRECT & $\begin{array}{c}\text { Regulation of } \\
\text { receptor activity }\end{array}$ & 2 & $8.5 \mathrm{E}-3$ \\
\hline 2. & GO_BP_DIRECT & $\begin{array}{c}\text { Anterior/posterior } \\
\text { pattern } \\
\text { specification }\end{array}$ & 2 & $7.4 \mathrm{E}-2$ \\
\hline 3. & GO_MF_DIRECT & $\begin{array}{c}\text { Poly(A) RNA } \\
\text { binding }\end{array}$ & 4 & $7.4 \mathrm{E}-2$ \\
\hline 4. & GO_MF_DIRECT & Protein binding & 12 & $8.0 \mathrm{E}-2$ \\
\hline
\end{tabular}

BP-biological process; FAT-functional annotation tool; MF-molecular function

TABLE 4: List of most significantly enriched pathways of DEGs as analyzed by KEGG pathway analysis

\begin{tabular}{|c|c|c|c|c|}
\hline S.NO. & PATHWAY & DESCRIPTION & $\begin{array}{c}\text { COUNT IN } \\
\text { GENE SET }\end{array}$ & $\begin{array}{c}\text { FALSE } \\
\text { DISCOVERY } \\
\text { RATE }\end{array}$ \\
\hline 1. & hsa04979 & Cholesterol metabolism & 8 of 48 & $2.79 \mathrm{e}-09$ \\
\hline 2. & hsa04934 & Cushing's syndrome & 8 of 153 & $6.90 \mathrm{e} 06$ \\
\hline 3. & hsa00310 & Lysine degradation & 6 of 59 & $6.90 \mathrm{e}-06$ \\
\hline 4. & hsa04919 & $\begin{array}{c}\text { Thyroid hormone signaling } \\
\text { pathway }\end{array}$ & 7 of 115 & $7.80 \mathrm{e}-06$ \\
\hline 5. & hsa00500 & Starch and sucrose metabolism & 5 of 33 & $7.80 \mathrm{e}-06$ \\
\hline 6. & hsa04137 & Mitophagy-animal & 5 of 63 & $9.95 \mathrm{e}-05$ \\
\hline 7. & hsa04211 & Longevity regulating pathway & 5 of 88 & 0.00040 \\
\hline 8. & hsa05215 & Prostate cancer & 5 of 97 & 0.00054 \\
\hline 9. & hsa04922 & Glucose signaling pathway & 5 of 100 & 0.00056 \\
\hline 10. & hsa04931 & Insulin resistance & 5 of 107 & 0.00068 \\
\hline 11. & hsa05203 & Viral carcinogenesis & 6 of 183 & 0.00071 \\
\hline 12. & hsa04152 & AMPK signaling pathway & 5 of 120 & 0.00096 \\
\hline
\end{tabular}




\begin{tabular}{|c|c|c|c|c|}
\hline 13. & hsa03320 & PPAR signaling pathway & 4 of 72 & 0.0017 \\
\hline 14. & hsa05166 & HTLV-I infection & 6 of 250 & 0.0029 \\
\hline 15. & hsa05202 & $\begin{array}{c}\text { Transcriptional misregulation in } \\
\text { cancer }\end{array}$ & 5 of 169 & 0.0036 \\
\hline 16. & hsa04975 & Fat digestion and absorption & 3 of 39 & 0.0040 \\
\hline 17. & hsa05200 & Pathways in cancer & 8 of 515 & 0.0042 \\
\hline 18. & hsa05167 & $\begin{array}{c}\text { Kaposi's sarcoma-associated } \\
\text { herpesvirus infection }\end{array}$ & 5 of 183 & 0.0043 \\
\hline 19. & hsa4330 & Notch signaling pathway & 3 of 48 & 0.0059 \\
\hline 20. & hsa05165 & Human papillomavirus infection & 6 of 317 & 0.0070 \\
\hline 21. & hsa05213 & Endometrial cancer & 3 of 58 & 0.0090 \\
\hline 22. & hsa04213 & $\begin{array}{l}\text { Longevity regulating pathway- } \\
\text { multiple species }\end{array}$ & 3 of 61 & 0.0099 \\
\hline 23. & hsa04151 & PI3K-Akt signaling pathway & 6 of 348 & 0.0099 \\
\hline 24. & hsa05161 & Hepatitis B & 4 of 142 & 0.0107 \\
\hline 25. & hsa05031 & Amphetamine addiction & 3 of 65 & 0.0107 \\
\hline 26. & hsa05224 & Breast cancer & 4 of 147 & 0.0111 \\
\hline 27. & hsa05206 & microRNAs in cancer & 4 of 149 & 0.0113 \\
\hline 28. & hsa04917 & Prolactin signaling pathway & 3 of 69 & 0.0113 \\
\hline 29. & hsa04218 & Cellular senescence & 4 of 156 & 0.0123 \\
\hline 30. & hsa05220 & Chronic myeloid leukemia & 3 of 76 & 0.0133 \\
\hline 31. & hsa04977 & Vitamin digestion and absorption & 2 of 24 & 0.0178 \\
\hline 32. & hsa05168 & Herpes simplex infection & 4 of 181 & 0.0188 \\
\hline 33. & hsa05169 & Epstein-barr virus infection & 4 of 194 & 0.0228 \\
\hline 34. & hsa05016 & Huntington's disease & 4 of 193 & 0.0228 \\
\hline 35. & hsa04916 & Melanogenesis & 3 of 98 & 0.0228 \\
\hline 36. & hsa04066 & HIF-1 signaling pathway & 3 of 98 & 0.0228 \\
\hline 37. & hsa05216 & Thyroid cancer & 2 of 37 & 0.0329 \\
\hline 38. & hsa04772 & Neutrophin signaling pathway & 3 of 116 & 0.0329 \\
\hline 39. & hsa04110 & Cell cycle & 3 of 123 & 0.0374 \\
\hline 40. & hsa05418 & $\begin{array}{l}\text { Fluid shear stress and } \\
\text { atherosclerosis }\end{array}$ & 3 of 133 & 0.0419 \\
\hline 41. & hsa05160 & Hepatis C & 3 of 131 & 0.0419 \\
\hline 42. & hsa04915 & Estrogen signaling pathway & 3 of 133 & 0.0419 \\
\hline 43. & hsa04910 & Insulin signaling pathway & 3 of 134 & 0.0419 \\
\hline 44. & Hsa04068 & FoxO signaling pathway & 3 of 130 & 0.0419 \\
\hline 45. & Hsa00071 & Fatty acid degradation & 2 of 44 & 0.0419 \\
\hline 46. & Hsa05030 & Cocaine addiction & 2 of 49 & 0.0427 \\
\hline 47. & Hsa01212 & Fatty acid metabolism & 2 of 48 & 0.0427 \\
\hline 48. & Hsa00280 & $\begin{array}{c}\text { Valine, leucine and isoleucine } \\
\text { degradation }\end{array}$ & 2 of 48 & 0.0427 \\
\hline 49. & Hsa04310 & Wnt signaling pathway & 3 of 143 & 0.0443 \\
\hline
\end{tabular}

TABLE 5: List of over represented pathway involved in Lung cancer using KEGG pathway analysis in STRING 


\begin{tabular}{|c|c|c|c|}
\hline PATHWAY & DESCRIPTION & $\begin{array}{c}\text { FALSE } \\
\text { DISCOVERY } \\
\text { RATE }\end{array}$ & $\begin{array}{c}\text { COLOR } \\
\text { REPRESENTA } \\
\text { TION }\end{array}$ \\
\hline hsa05203 & Viral carcinogenesis & 0.00071 & Dark purple \\
\hline hsa04152 & AMPK signaling pathway & 0.00096 & Pale yellow \\
\hline hsa03320 & PPAR signaling pathway & 0.0017 & Cyan \\
\hline hsa05200 & Pathways in cancer & 0.0042 & Dark green \\
\hline hsa4330 & Notch signaling pathway & 0.0059 & Pink \\
\hline hsa04151 & PI3K-Akt signaling & 0.0099 & Yellow \\
\hline hsa05206 & mathway & & Green \\
\hline hsa04066 & HIF-1 signaling pathway & 0.0228 & Dark blue \\
\hline hsa00280 & Valine, leucine and & 0.0427 & Purple \\
& isoleucine degradation & & Red \\
\hline hsa04310 & Wnt signaling pathway & 0.0423 & \\
\hline
\end{tabular}

Table 6: Top 10 Hub genes with their ranks and scores respectively

\begin{tabular}{|c|c|c|}
\hline Rank & Name & Score \\
\hline 1 & EP300 (E1A Binding Protein P300) & 29 \\
\hline 2 & TP53 (Tumor protein 53) & 25 \\
\hline 3 & KAT2B(K(lysine) acetyltransferase 2B) & 24 \\
\hline 4 & HDAC1 (Histone Deacetylase 1) & 22 \\
\hline 5 & SIRT1 (sirtuin 1) & 17 \\
\hline 6 & KMT2A(Lysine Methyltransferase 2A) & 16 \\
\hline 6 & ASH2L (histone lysine methyltransferasecomplex & 16 \\
\hline 6 & subunit) & 16 \\
\hline 6 & SETD1B (SET Domain Containing 1B) & 16 \\
\hline 10 & SETD1A (SET Domain Containing 1A) & 15 \\
\hline
\end{tabular}

Table 7: Top 3 modules from PPI network along with the genes involved

\begin{tabular}{|c|l|l|}
\hline MODULES & PATHWAY INVOLVED & GENES INVOLVED \\
\hline \multirow{2}{*}{1.} & Viral carcinogenesis & KAT2B,EP300,RELA,CREB1,TP53 \\
\cline { 2 - 3 } & Pathways in cancer & HDAC1,TP53,EP300 \\
& & \\
\hline
\end{tabular}




\begin{tabular}{|l|l|l|}
\hline \multirow{2}{*}{2.} & Notch signaling pathway & KAT2B,HDAC1,EP300 \\
\cline { 2 - 3 } & MicroRNAs in cancer & HDAC1,EP300,TP53 \\
\cline { 2 - 3 } & Wnt signaling pathway & TP53,EP300 \\
\hline & Viral carcinogenesis & RELA,CREB1 \\
\cline { 2 - 3 } & AMPK signaling pathway & SIRT1,FOXO3,CREB1 \\
\cline { 2 - 3 } & PPAR signaling pathway & ACADM,APOA1,APOA2,APOC3 \\
\cline { 2 - 3 } & Pathways in cancer & NCOA3,NCOA1,RELA,HIF1A \\
\cline { 2 - 3 } & PI3K-Akt signaling pathway & RELA,FOXO3,CREB1 \\
\cline { 2 - 3 } & HIF-1 signaling pathway & HIF1A,RELA \\
\cline { 2 - 3 } & $\begin{array}{l}\text { Valine, leucine and isoleucine } \\
\text { degradation }\end{array}$ & ACAA2,ACADM \\
\hline \multirow{2}{*}{3.} & AMPK signaling pathway & GYS1,GYS2 \\
\cline { 2 - 3 } & PI3K-Akt signaling pathway & GYS1,GYS2 \\
\cline { 2 - 3 } & Pathways in cancer & TCF7L2 \\
\cline { 2 - 3 } & Wnt signaling pathway & TCF72 \\
\hline
\end{tabular}




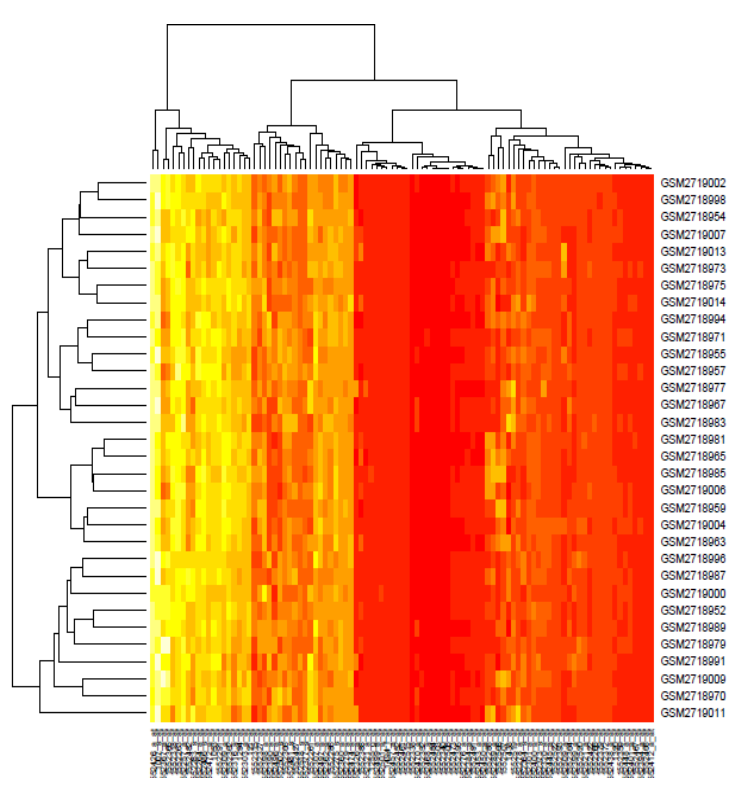

a)

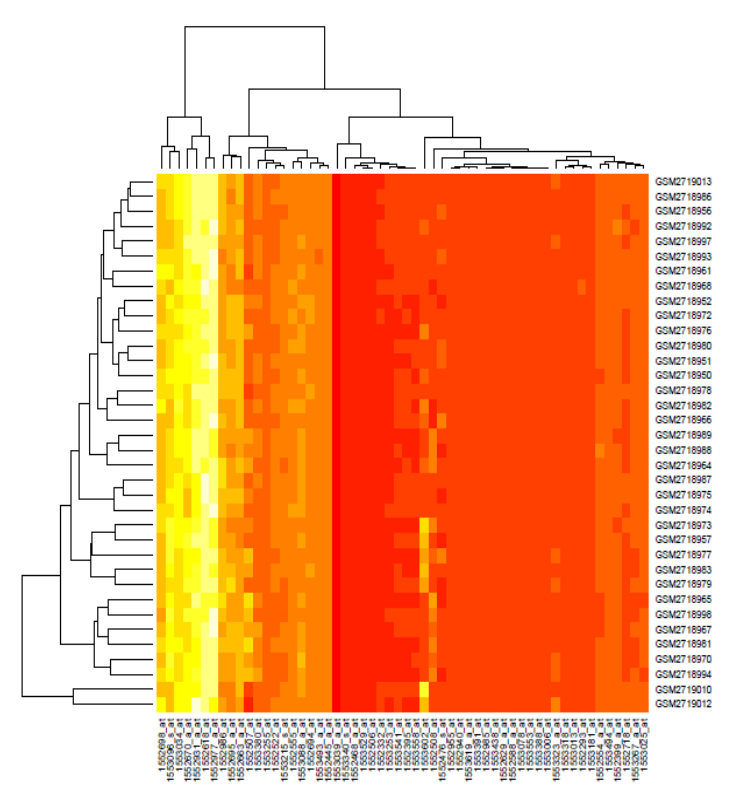

c)

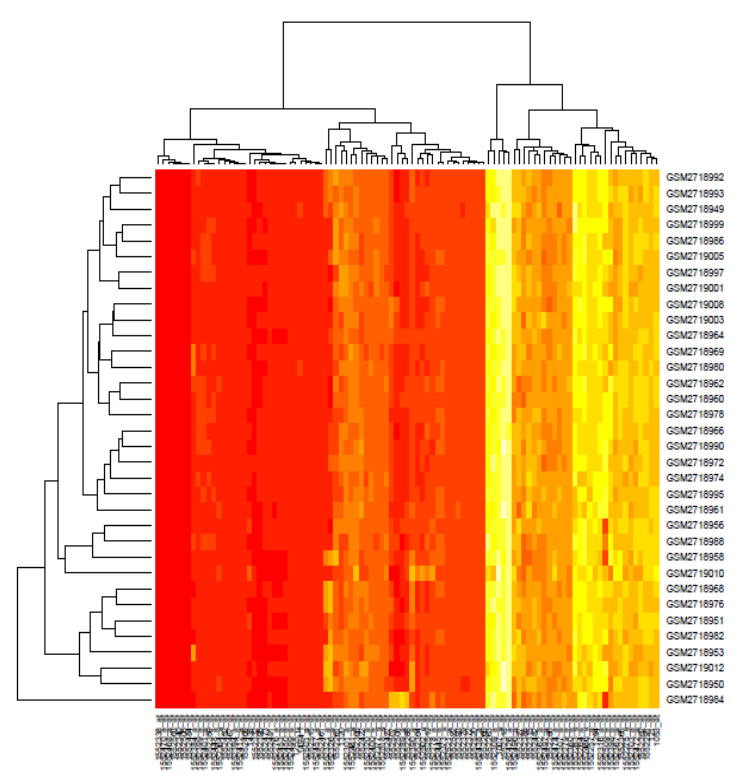

b)

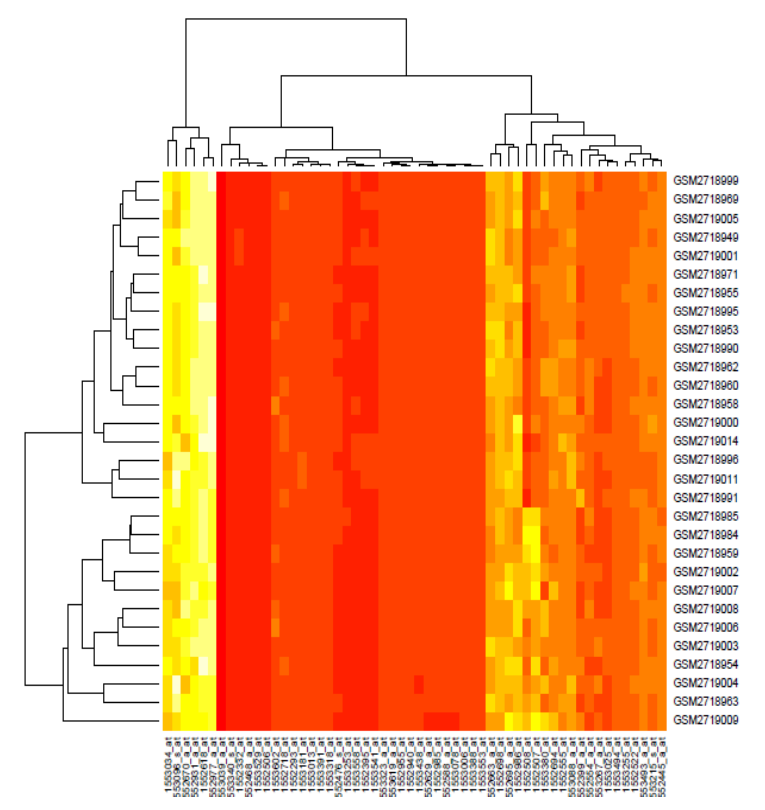

d) 


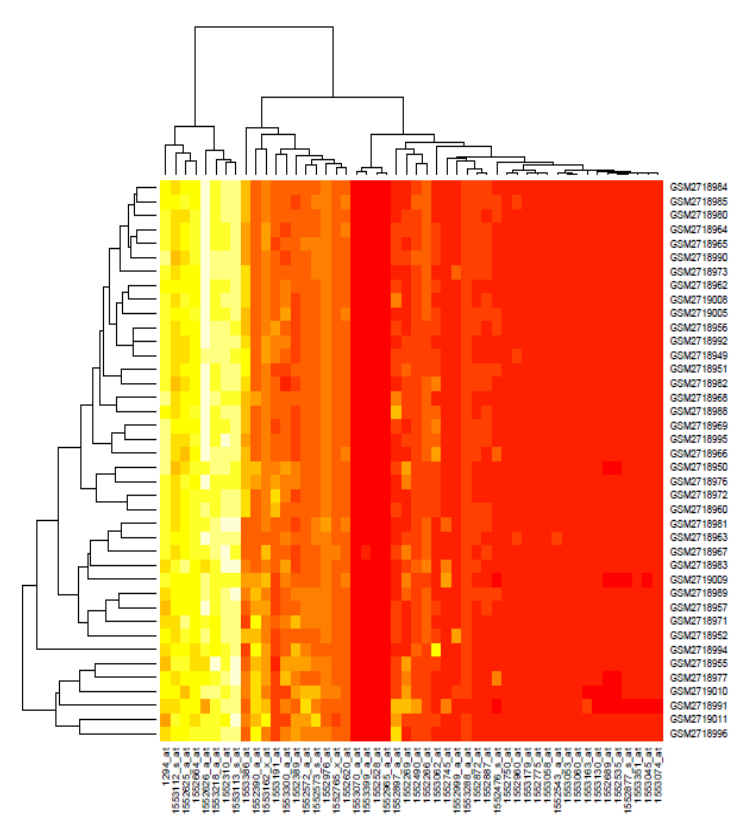

e)

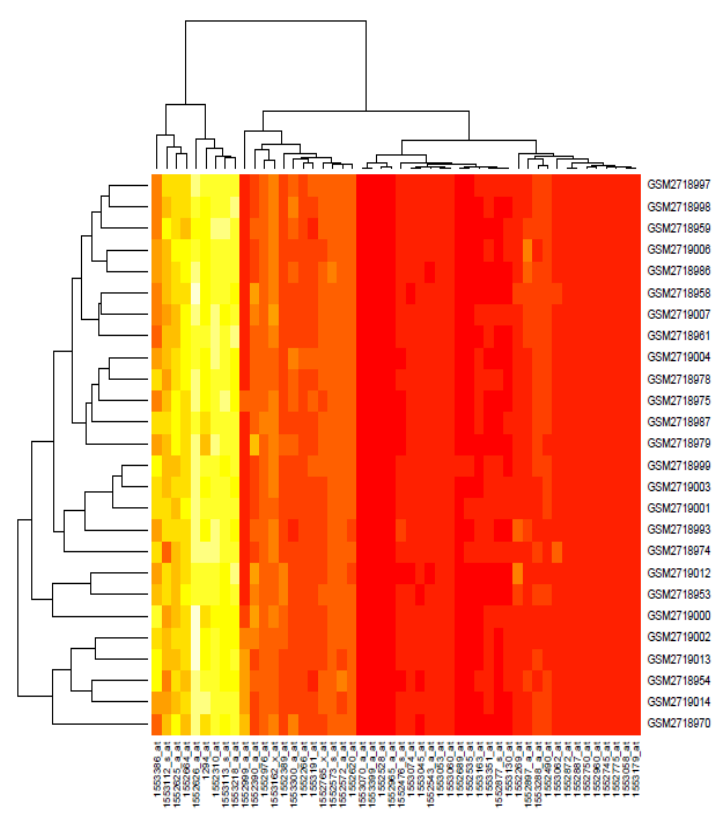

f)

Figure 1: heat maps showing the gene expression levels a) tumor present b) tumor absent c) stage 1 d) stage 2 e) dead status f) alive status. 


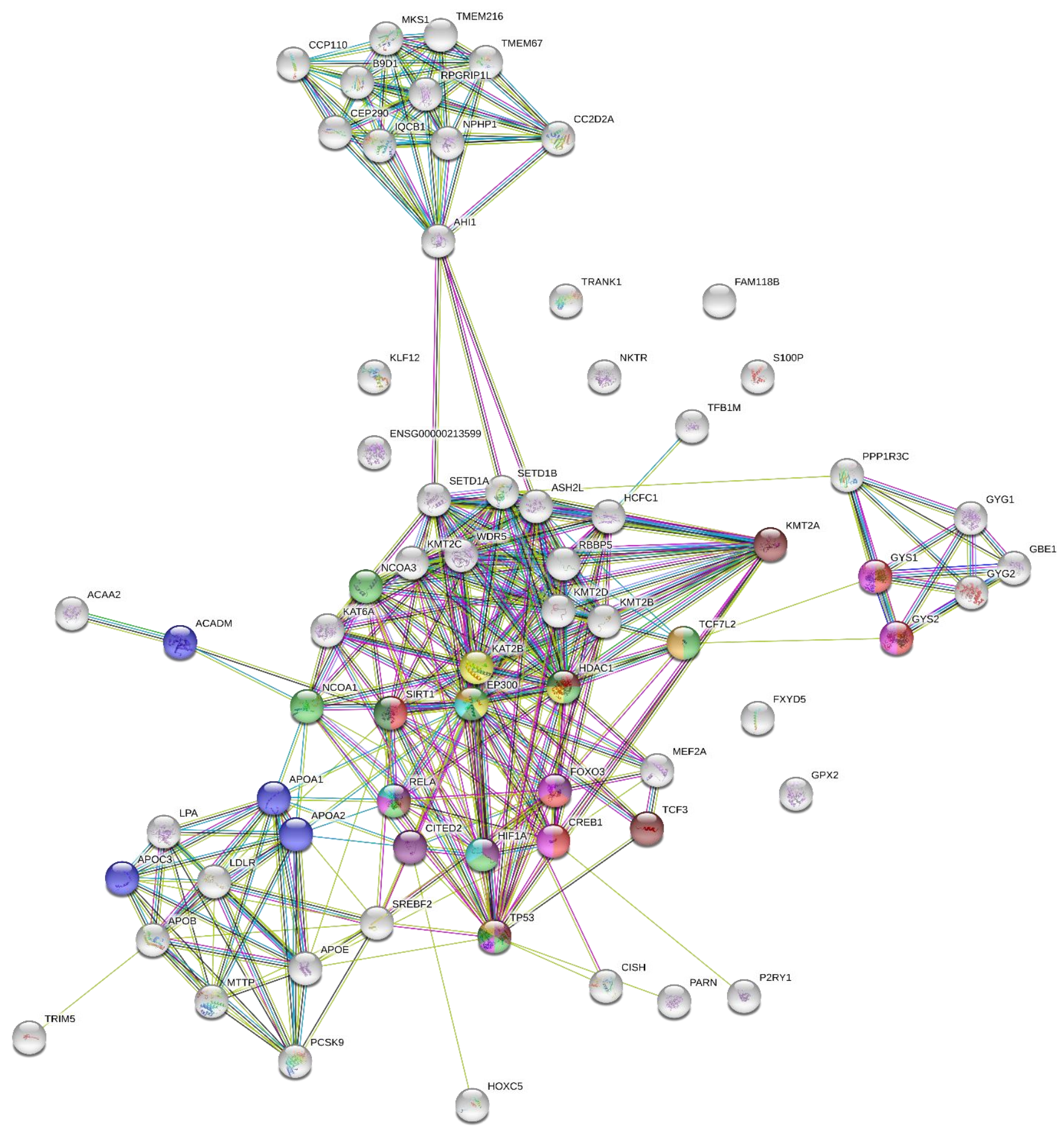

Figure 2: PPI network of DEGs identified by STRING 


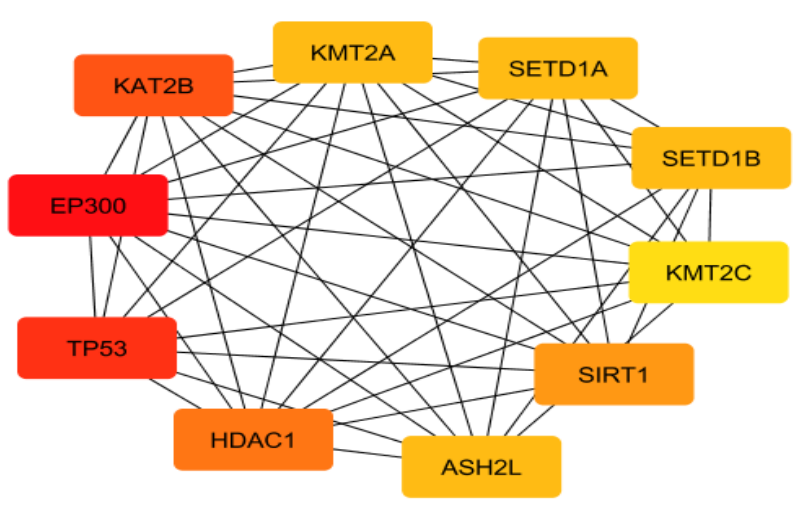

\begin{tabular}{|l|l|}
\hline Rank & $\begin{array}{l}\text { Node } \\
\text { EP300 }\end{array}$ \\
\hline 1 & TP53 \\
\hline 2 & KAT2B \\
\hline 3 & HDAC 1 \\
\hline 5 & SIRT 1 \\
\hline 6 & KMT2A \\
\hline 6 & ASH2L \\
\hline 6 & SETD 1B \\
\hline 6 & SETD 1A \\
\hline 10 & KMTT2C \\
\hline
\end{tabular}

Figure 3: Top 10 Hub Genes with highest degree of interaction in Lung cancer as analyzed by CYTOHUBBA plugin of Cytoscape.

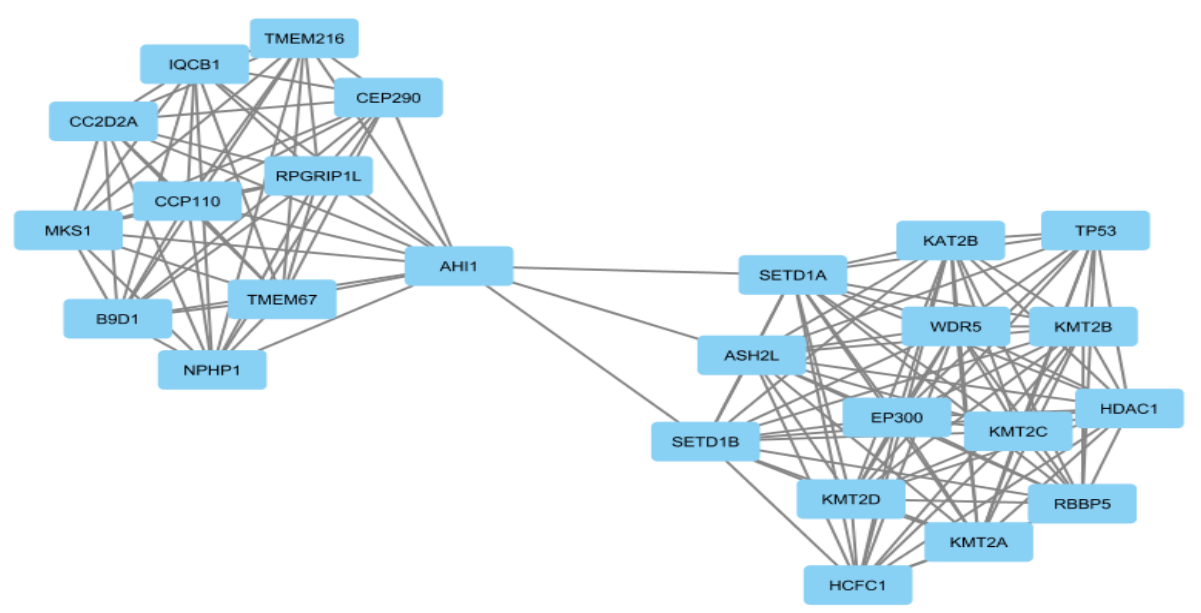

(A) 


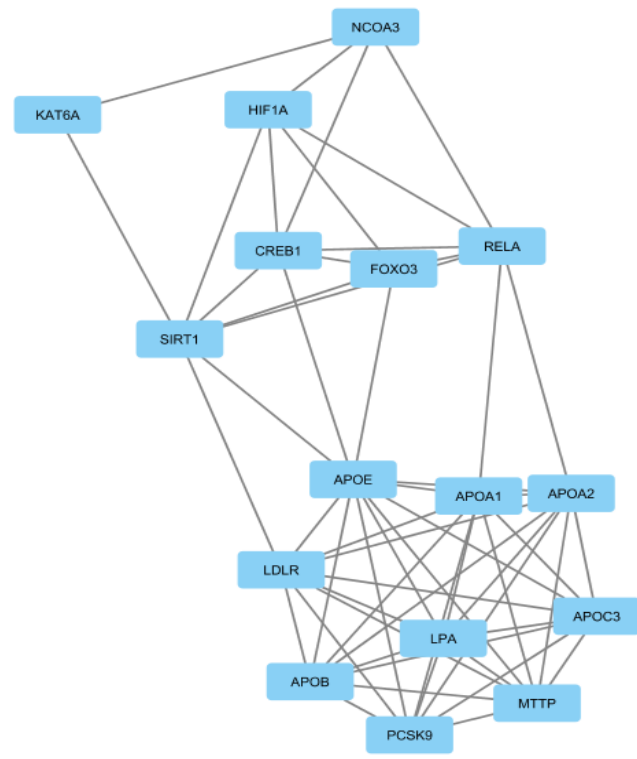

(B)

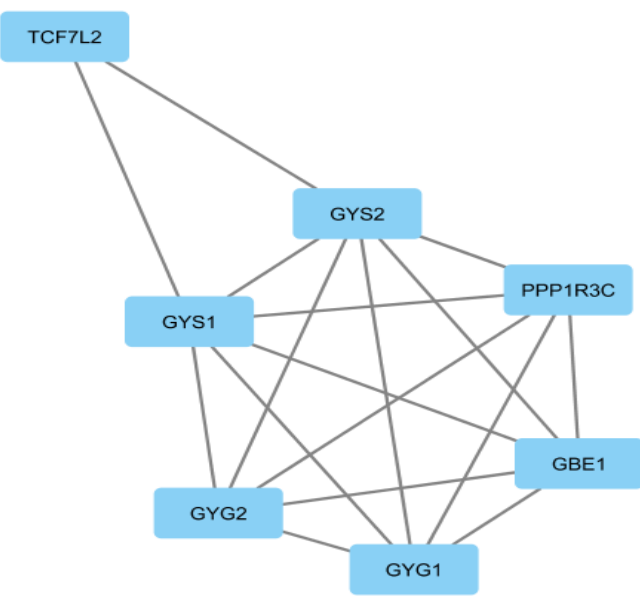

(C)

Figure 4: Top 3 modules of PPI networks. Nodes and links show human proteins and their PPI. A) Enriched pathway of module A; B) Enriched pathway of module B; C) Enriched pathway of module $C$

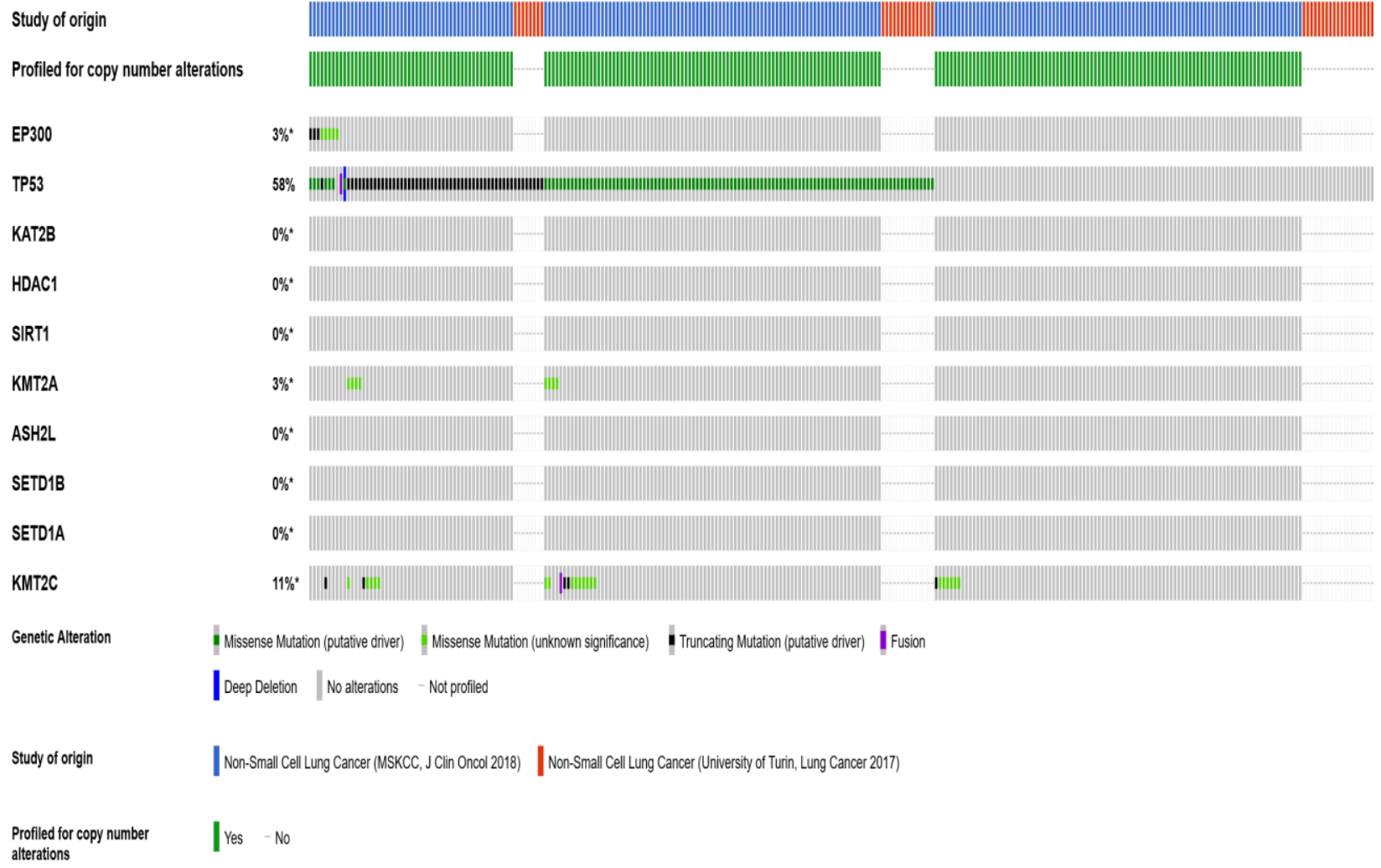

Figure 5: Oncoprint of the hub genes analyzed by cBioPortal tool OF TCGA database. 


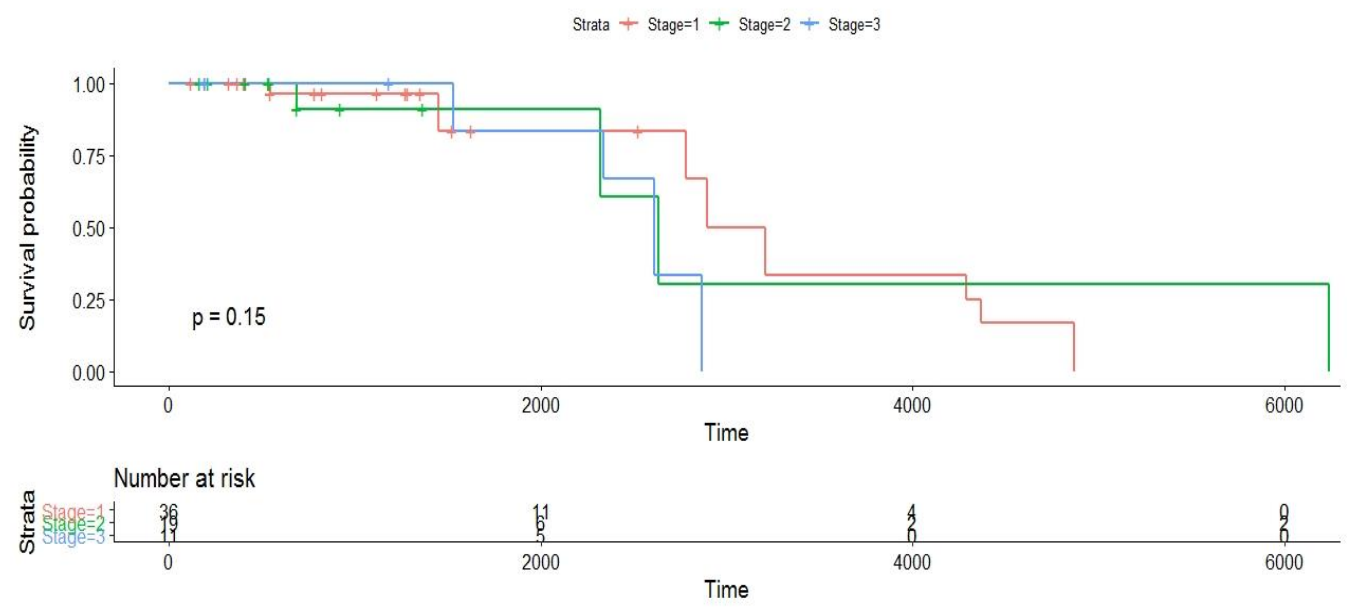

Figure 6: Stage wise survival of lung cancer patients among AAs. 